\title{
A GEOMETRIC APPROACH TO STRUCTURAL MODEL MATCHING BY OUTPUT FEEDBACK IN LINEAR IMPULSIVE SYSTEMS
}

\author{
ELENA ZATTONI ${ }^{a}$
}

\author{
${ }^{a}$ Department of Electrical, Electronic, and Information Engineering "G. Marconi" \\ Alma Mater Studiorum • University of Bologna, Viale Risorgimento 2, 40136 Bologna, Italy \\ e-mail: elena.zattoni@unibo.it
}

\begin{abstract}
This paper provides a complete characterization of solvability of the problem of structural model matching by output feedback in linear impulsive systems with nonuniformly spaced state jumps. Namely, given a linear impulsive plant and a linear impulsive model, both subject to sequences of state jumps which are assumed to be simultaneous and measurable, the problem consists in finding a linear impulsive compensator that achieves exact matching between the respective forced responses of the linear impulsive plant and of the linear impulsive model, by means of a dynamic feedback of the plant output, for all the admissible input functions and for all the admissible sequences of jump times. The solution of the stated problem is achieved by reducing it to an equivalent problem of structural disturbance decoupling by dynamic feedforward. Indeed, this latter problem is formulated for the so-called extended linear impulsive system, which consists of a suitable connection between the given plant and a modified model. A necessary and sufficient condition for the solution of the structural disturbance decoupling problem is first shown. The proof of sufficiency is constructive, since it is based on the synthesis of the compensator that solves the problem. The proof of necessity is based on the definition and the geometric properties of the unobservable subspace of a linear impulsive system subject to unequally spaced state jumps. Finally, the equivalence between the two structural problems is formally established and proven.
\end{abstract}

Keywords: linear impulsive systems, hybrid systems, model matching, disturbance decoupling, geometric approach, structural properties.

\section{Introduction}

Linear impulsive systems are a special class of hybrid dynamical systems-also known as hybrid dynamical systems with state jumps-characterized by a continuous-time linear behavior interlaced with abrupt state changes which occur at isolated time instants. The continuous-time linear dynamics is called flow $d y$ namics, while the state discontinuities give rise to the so-called jump dynamics. Hybrid systems, in general, and linear impulsive systems, in particular, have drawn a considerable amount of research effort during the last decades, as is documented by the broad literature on the subject-see, e.g., the books by Van der Schaft and Schumacher (2000), Savkin and Evans (2002), Li et al. (2005), Haddad et al. (2006) and Goebel et al. (2012), as well as the collections edited by Engell et al. (2002) and Djemai and Deefort (2015).

Concerning linear impulsive systems, these have proven to be very effective especially in modeling complex phenomena-like, for instance, collisions-as well as composite systems, such as, e.g., mechatronic systems including both analog and digital components. The reader may again refer to the above-mentioned monographs for more details about specific applications and case studies. Concerning the state of the art of the methodologies developed to handle this class of dynamical systems, it has to be acknowledged that, nowadays, several control and observation problems have already been formulated and studied in the context of linear impulsive systems. More specifically, problems that have recently been tackled concern state estimation (Medina and Lawrence, 2009; Conte et al., 2017), linear quadratic control (Kouhi et al., 2013; Carnevale et al., 2014b), disturbance decoupling (Conte et al., 2015a; Perdon et al., 2016b), output regulation (Medina and Lawrence, 2006; Zattoni et al., 2015; 2017b; 2017a; Carnevale et al., 2016), and model matching (Zattoni, 
2016b). Nevertheless, the analysis of several aspects of the problems mentioned above remains to be deepened further and, therefore, the research in the field is still truly active.

As to model matching, this is a classical problem of control theory. In fact, the problem of compensating a given plant in such a way that its forced response matches that of a given model for all the admissible input signals was first stated and solved by state feedback for linear time-invariant systems in the early 1970s (Wolovich, 1972; Morse, 1973). More recently, a solution to the model matching problem by output dynamic feedback in multivariable linear systems has been obtained by means of the geometric approach (Marro and Zattoni, 2002; 2005). As a counterpart of the geometric approach to model matching in multivariable linear systems, it is worth mentioning the polynomial approach (Kaczorek, 1982).

Since the earlier works, the model matching problem has been reformulated for more general classes of dynamical systems like, e.g., nonlinear systems (Noldus, 1987; Moog et al., 1991; Lin and Tsai, 1999), descriptor systems (Kučera, 1992), time-delay and uncertain systems (Shyu and Chen, 1995; Ni et al., 2001; Wu, 2004; Wang et al., 2009), periodic systems (Colaneri and Kučera, 1997), large-scale systems (Shioemaru and Wu, 2001; Hua and Ding, 2011), Markovian jump linear systems (Wang et al., 2012), switching systems (Zattoni et al., 2014b; Conte et al., 2014; Perdon et al., 2016a; 2016c), and, as previously mentioned, linear impulsive systems (Zattoni, 2016b). At this point, it is worth to emphasize that the wide and long lasting interest in model matching problems is motivated not only by the impact that the solution of this kind of problems has on applications, but also by the fact that model matching represents a basic methodological tool to master more complex control problems (see, e.g., Choe and Kim, 2002; Pascual et al., 2008; Rajasekaran et al., 2009; Zakharov et al., 2013; 2015).

In this framework, the contribution of the present work consists in providing a necessary and sufficient condition for the existence of a solution to the problem of structural model matching in linear impulsive systems. In particular, the linear impulsive systems dealt with are assumed to be subject to jump time sequences whose elements are not equally spaced. Such jump time sequences are assumed not to be known a-priori, but to be measurable. A standing assumption throughout the discussion is also that any finite time interval contains only a finite number of jump times, which ensures that Zeno behaviors are excluded. The control scheme considered is based on output feedback, through a compensator which is itself a linear impulsive system subject to a jump time sequence which is the same as that of the linear impulsive plant and model.
With respect to the earlier paper (Zattoni, 2016b), a major difference concerns the structure of the linear impulsive plant. In the work of Zattoni (2016b), the linear impulsive plant exhibits a feedthrough term from the control input to the output, while here that term is absent. This implies that different geometric tools are used to handle the problem. In particular, output-nulling controlled invariant subspaces for linear impulsive systems with the feedthrough term are replaced by controlled invariant subspaces contained in the null space of the output map for linear impulsive systems without the feedthrough term. Leaving output-nulling controlled invariant subspaces out of consideration implies, on the one hand, that some heavy technicalities are not required anymore. On the other hand, this fact leaves space for a deeper treatment of some theoretical aspects. In particular, in this work, the proof of the necessary and sufficient condition for solvability of the equivalent structural disturbance decoupling problem is based on the concept of unobservable subspace for a linear impulsive system.

As to the methodological background, this hinges on the geometric approach (Wonham, 1985; Basile and Marro, 1992), which has been extended to cope with linear impulsive systems by various authors (Medina and Lawrence, 2006; Lawrence, 2014; 2015; Carnevale et al., 2014a). However, since linear impulsive systems may exhibit a different structure (e.g., jump dynamics may be either free or controlled, the output equation may be either only one, valid for both the flow and the jump dynamics, or may be twofold, so as to separately consider the outputs for the flow and the jump dynamics, etc.), it is preferred herein to refer to the notions introduced in some of our previous papers (e.g., Zattoni et al., 2017b), since they address linear impulsive systems with the same characteristics as those considered herein. On a last note, it is worth mentioning that the geometric approach has proven to be very effective also in mastering control problems stated for other classes of hybrid systems, such as switched linear systems (Zattoni et al., 2013a; 2013b; Zattoni, 2014; Conte et al., 2013; Zattoni and Marro, 2013b).

The remainder of the work is organized as follows. The problem of structural model matching by output feedback for linear impulsive systems is stated in Section 2. The problem of structural disturbance decoupling by feedforward is formulated in Section 3 . In Section 4, the geometric notions needed to approach the problems in question are set forth. Section 5 shows a necessary and sufficient condition for solvability of the disturbance decoupling problem. Section 6 formally establishes the equivalence between the two problems and, consequently, between the respective solutions. Section 7 presents some concluding remarks. 
Notation. The symbols $\mathbb{R}$ and $\mathbb{R}^{+}$stand for the sets of real numbers and nonnegative real numbers, respectively. Matrices and linear maps are denoted by slanted upper-case letters, like $A$. The image and the kernel of $A$ are denoted by $\operatorname{Im} A$ and $\operatorname{Ker} A$, respectively. The inverse of a nonsingular square matrix $A$ is denoted by $A^{-1}$. Vector spaces and subspaces are denoted by calligraphic letters, like $\mathcal{V}$. The notation $B^{-1} \mathcal{V}$, where $B$ is not necessarily an invertible linear map and $\mathcal{V}$ is a subspace of a vector space whose dimension is consistent with $B$, stands for the inverse image through $B$ of the subspace $\mathcal{V}$. The notation $\mathcal{V} \oplus \mathcal{W}=\mathcal{X}$, where $\mathcal{V}$ and $\mathcal{W}$ are subspaces of the vector space $\mathcal{X}$, stands for the direct sum of $\mathcal{V}$ and $\mathcal{W}$, namely, $\mathcal{V}+\mathcal{W}=\mathcal{X}$ and $\mathcal{V} \cap \mathcal{W}=\{0\}$. Let $A: \mathcal{X} \rightarrow \mathcal{X}$ denote a linear map from a vector space $\mathcal{X}$ to the same vector space $\mathcal{X}$, and let $\mathcal{C}$ denote a subspace of $\mathcal{X}$. The symbol max $\mathcal{J}(A, \mathcal{C})$ stands for the maximal $A$-invariant subspace contained in $\mathcal{C}$. The symbol $I$ denotes an identity matrix of appropriate dimension.

\section{Problem statement: Structural model matching by output feedback}

In order to formally describe linear impulsive systems, an appropriate definition of the so-called hybrid time domain is required in the first place. In the general case of nonuniformly spaced state jumps, the hybrid time domain consists of an interlaced sequence of continuous time intervals with various lengths and isolated time instants which correspond to the times when the state shows discontinuities. The number of the discontinuity points is assumed to be finite in any finite time interval or, equivalently, the set of the discontinuity points is assumed not to show any accumulation point. This hypothesis guarantees that the linear impulsive systems defined on this time domain do not exhibit Zeno behaviours. In more formal terms, the set of the discontinuity points of the time axis is defined as a finite or countably infinite set of strictly increasing points of the nonnegative real axis $\mathbb{R}^{+}$and is denoted by $\mathcal{T}=\left\{t_{0}, t_{1}, \ldots\right\}$. The set of all the sequences $\mathcal{T}$ of jump times satisfying the constraint of presenting no accumulation points is denoted by $\mathscr{T}$.

Secondly, in order to formulate the problem of structural model matching by output feedback for linear impulsive systems with nonuniformly spaced state jumps, a plant $\Sigma_{P}$, a model $\Sigma_{M}$, and a compensator $\Sigma_{C}$ must be defined. The linear impulsive system with nonuniformly spaced state jumps $\Sigma_{P}$ is defined by

$$
\Sigma_{P} \equiv\left\{\begin{aligned}
\dot{x}_{P}(t)=A_{P} x_{P}(t)+ & B_{P} u(t), \\
& \text { with } t \in \mathbb{R}^{+} \backslash \mathcal{T}, \\
x_{P}(t)=J_{P} x_{P}\left(t^{-}\right), & \text {with } t=t_{k}, t_{k} \in \mathcal{T}, \\
y_{P}(t)=C_{P} x_{P}(t), & \text { with } t \in \mathbb{R}^{+},
\end{aligned}\right.
$$

where $x_{P} \in \mathcal{X}_{P}=\mathbb{R}^{n_{P}}$ is the state, $u \in \mathbb{R}^{p}$ is the control input, and $y_{P} \in \mathbb{R}^{q}$ is the output, with $p, q \leq n_{P}$. The matrices $A_{P}, B_{P}, J_{P}$, and $C_{P}$ have suitable dimensions and constant real entries. In particular, $B_{P}$ and $C_{P}$ are assumed to be full rank. The set of the admissible control input functions $u(t)$, with $t \in \mathbb{R}^{+}$, is assumed to be the set of all piecewise-continuous functions with values in $\mathbb{R}^{p}$. The differential state equation governs the so-called flow dynamics. The symbol $x_{P}\left(t^{-}\right)$stands for $\lim _{\tau \rightarrow 0^{+}} x_{P}(t-\tau)$. The algebraic state equation governs the so-called jump dynamics. Hence, according to the linear impulsive structure of $\Sigma_{P}$, the state trajectory $x_{P}(t)$ in the time interval $\left[0, t_{0}\right)$ is the solution of the differential equation, with given initial state $x_{P}(0)$ and input function $u(t)$, with $t \in\left[0, t_{0}\right)$. The state $x_{P}(t)$, with $t=t_{k}$ and $t_{k} \in \mathcal{T}$, is the image of $x_{P}\left(t^{-}\right)$through $J_{P}$. The state trajectory $x_{P}(t)$ in the time interval $\left[t_{k} t_{k+1}\right)$, with $t_{k} \in \mathcal{T}$, is the solution of the differential equation, with given initial state $x_{P}\left(t_{k}\right)$ and input function $u(t)$, with $t \in\left[t_{k} t_{k+1}\right)$. Hence, whenever the linear map $J_{P}$, governing the plant jump dynamics, is different from the identity, it generates a discontinuity of the state evolution at each time $t_{k}$ belonging to the set $\mathcal{T}$.

The linear impulsive model with nonuniformly spaced state jumps $\Sigma_{M}$ is defined by

$$
\Sigma_{M} \equiv \begin{cases}\dot{x}_{M}(t)=A_{M} x_{M}(t)+ & B_{M} d(t), \\ & \text { with } t \in \mathbb{R}^{+} \backslash \mathcal{T}, \\ x_{M}(t)=J_{M} x_{M}\left(t^{-}\right), & \text {with } t=t_{k}, t_{k} \in \mathcal{T}, \\ y_{M}(t)=C_{M} x_{M}(t), & \text { with } t \in \mathbb{R}^{+},\end{cases}
$$

where $x_{M} \in \mathbb{R}^{n_{M}}$ is the state, $d \in \mathbb{R}^{q}$ is the input, and $y_{M} \in \mathbb{R}^{q}$ is the output. $A_{M}, B_{M}, J_{M}$, and $C_{M}$ are constant real matrices with suitable dimensions. In particular, $B_{M}$ and $C_{M}$ are assumed to be full rank. The set of the admissible input functions $d(t)$, with $t \in \mathbb{R}^{+}$, is assumed to be the set of all piecewise-continuous functions with values in $\mathbb{R}^{q}$.

The to-be-designed linear impulsive compensator with nonuniformly spaced state jumps $\Sigma_{C}$ is defined by

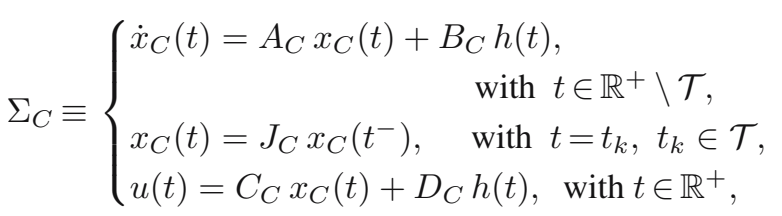

where $x_{C} \in \mathbb{R}^{n_{C}}$ is the state, $h \in \mathbb{R}^{q}$ is the input, and $u \in \mathbb{R}^{p}$ is the output. $A_{C}, B_{C}, J_{C}, C_{C}$, and $D_{C}$ are constant real matrices of suitable dimensions. The set of the admissible input functions $h(t)$, with $t \in \mathbb{R}^{+}$, is assumed to be the set of all piecewise-continuous functions with values in $\mathbb{R}^{q}$. It is worth noting that the structure of the linear impulsive compensator $\Sigma_{C}$ presented above presupposes that the jump time instants of the plant and of the model, which are assumed to be simultaneous, are known (i.e., measurable). 


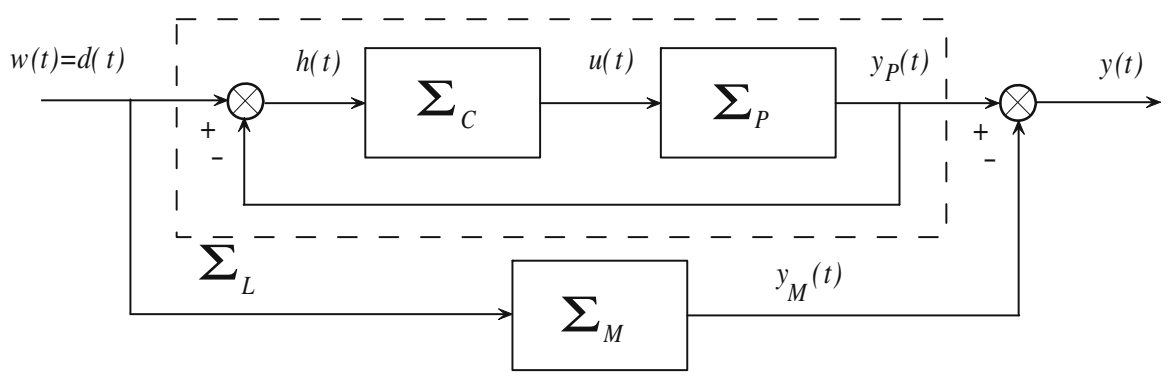

Fig. 1. Block diagram for structural model matching by output feedback.

Since, in the model matching control scheme considered in this section, the linear impulsive compensator $\Sigma_{C}$ is assumed to act as an output-feedback compensator, the input $h(t)$ is taken as the difference between the new input $w(t)$, which is assumed to belong to the set of all piecewise-continuous functions with values in $\mathbb{R}^{q}$, and the feedback signal, which consists of the plant output $y_{P}(t)$, namely,

$$
h(t)=w(t)-y_{P}(t), \text { with } t \in \mathbb{R}^{+} .
$$

In this context, the problem of structural model matching by output feedback for linear impulsive systems with nonuniformly spaced state jumps can be formulated as follows.

Problem 1. (Structural model matching by output feedback) Let the linear impulsive system $\Sigma_{P}$ and the linear impulsive model $\Sigma_{M}$ be given. Find an output-feedback linear impulsive compensator $\Sigma_{C}$ such that the closed-loop linear impulsive system $\Sigma_{L}$, defined by

$$
\Sigma_{L} \equiv \begin{cases}\dot{x}_{L}(t)=A_{L} x_{L}(t)+ & B_{L} w(t), \\ & \text { with } t \in \mathbb{R}^{+} \backslash \mathcal{T}, \\ x_{L}(t)=J_{L} x_{L}\left(t^{-}\right), & \text {with } t=t_{k}, t_{k} \in \mathcal{T}, \\ y_{P}(t)=C_{L} x_{L}(t), & \text { with } t \in \mathbb{R}^{+},\end{cases}
$$

where

$$
\begin{aligned}
A_{L} & =\left[\begin{array}{cc}
A_{P}-B_{P} D_{C} C_{P} & B_{P} C_{C} \\
-B_{C} C_{P} & A_{C}
\end{array}\right], \\
B_{L} & =\left[\begin{array}{c}
B_{P} D_{C} \\
B_{C}
\end{array}\right], \\
J_{L} & =\left[\begin{array}{cc}
J_{P} & 0 \\
0 & J_{C}
\end{array}\right], \\
C_{L} & =\left[\begin{array}{ll}
C_{P} & 0
\end{array}\right],
\end{aligned}
$$

satisfies the requirement that its output $y_{P}(t)$ is equal to the model output $y_{M}(t)$, for all $t \in \mathbb{R}^{+}$, or, equivalently, the difference

$$
y(t)=y_{P}(t)-y_{M}(t), \quad \text { with } t \in \mathbb{R}^{+},
$$

is equal to zero, when the respective inputs are taken equal, i.e.,

$$
w(t)=d(t), \quad \text { with } t \in \mathbb{R}^{+},
$$

and the respective initial states are zero, for all the admissible input functions $d(t)$, with $t \in \mathbb{R}^{+}$, and for all the admissible sequences of jump times $\mathcal{T} \in \mathscr{T}$.

The system interconnection considered in Problem 1 is illustrated in the block diagram of Fig. 1

\section{Problem statement: Structural disturbance decoupling by feedforward}

The purpose of this section is to introduce the problem of structural disturbance decoupling by feedforward for linear impulsive systems with nonuniformly spaced state jumps. In general, disturbance decoupling by a feedforward action is a viable option when the disturbance is (at least) accessible for measurement-a detailed discussion of this topic for the case of linear time-invariant systems can be found, e.g., in the work of Zattoni (2007). In particular, the interest in considering structural disturbance decoupling by feedforward in the context of model matching by output feedback for linear impulsive systems with nonuniformly spaced state jumps lies in the fact that the solution of the structural model matching problem previously stated can be achieved through the solution of a structural disturbance decoupling problem by feedforward, formulated for a suitably defined linear impulsive system, as will be shown in the next sections.

Hence, the so-called modified linear impulsive model with nonuniformly spaced state jumps, from now on denoted by $\tilde{\Sigma}_{M}$, is derived by closing a positive unit output feedback on the flow dynamics of the original linear impulsive model $\Sigma_{M}$. Namely, by taking

$$
d(t)=v(t)+y_{M}(t), \quad \text { with } t \in \mathbb{R}^{+},
$$

where the new input $v(t)$, with $t \in \mathbb{R}^{+}$, is assumed to belong to the set of all piecewise-continuous functions 


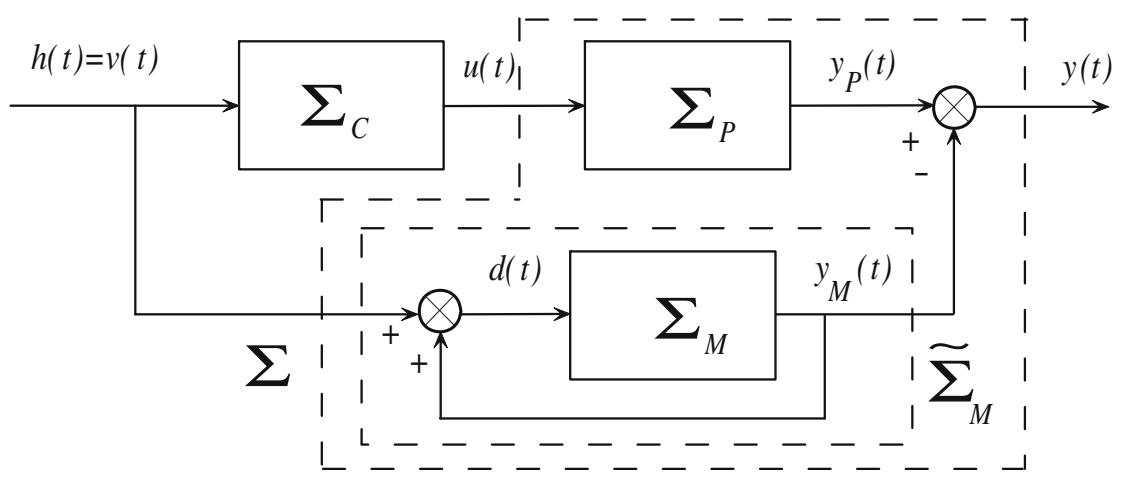

Fig. 2. Block diagram for structural disturbance decoupling by feedforward.

with values in $\mathbb{R}^{q}$, one gets

$\tilde{\Sigma}_{M} \equiv\left\{\begin{aligned} & \dot{x}_{M}(t)=\left(A_{M}+B_{M} C_{M}\right) x_{M}(t)+B_{M} v(t), \\ & \text { with } t \in \mathbb{R}^{+} \backslash \mathcal{T}, \\ & x_{M}(t)=J_{M} x_{M}\left(t^{-}\right), \text {with } t=t_{k}, t_{k} \in \mathcal{T}, \\ & y_{M}(t)=C_{M} x_{M}(t), \text { with } t \in \mathbb{R}^{+} .\end{aligned}\right.$

Moreover, the so-called extended linear impulsive system with nonuniformly spaced state jumps, henceforth denoted by $\Sigma$, is defined as the output-difference connection between the given linear impulsive system $\Sigma_{P}$ and the modified linear impulsive model $\tilde{\Sigma}_{M}$. Consequently, by defining $y(t)$ as in (1), one obtains that the linear impulsive system $\Sigma$ is described by

$$
\Sigma \equiv\left\{\begin{aligned}
& \dot{x}(t)=A x(t)+B u(t)+H v(t), \\
& \text { with } t \in \mathbb{R}^{+} \backslash \mathcal{T}, \\
& x(t)=J x\left(t^{-}\right), \text {with } t=t_{k}, t_{k} \in \mathcal{T}, \\
& y(t)=C x(t), \text { with } t \in \mathbb{R}^{+},
\end{aligned}\right.
$$

where

$$
\begin{aligned}
A & =\left[\begin{array}{cc}
A_{P} & 0 \\
0 & A_{M}+B_{M} C_{M}
\end{array}\right], \\
B & =\left[\begin{array}{c}
B_{P} \\
0
\end{array}\right], \\
H & =\left[\begin{array}{c}
0 \\
B_{M}
\end{array}\right], \\
J & =\left[\begin{array}{cc}
J_{P} & 0 \\
0 & J_{M}
\end{array}\right], \\
C & =\left[\begin{array}{ll}
C_{P} & -C_{M}
\end{array}\right] .
\end{aligned}
$$

The state space of $\Sigma$ is denoted by $\mathcal{X}$, i.e., $\mathcal{X}=\mathbb{R}^{n}$, where $n=n_{P}+n_{M}$.

Furthermore, it is worth noting that the feedforward compensation scheme considered herein presupposes that the linear impulsive compensator $\Sigma_{C}$ performs a feedforward action. This means that $\Sigma_{C}$ is fed by the same input $v(t)$ which is applied to the modified linear impulsive model $\tilde{\Sigma}_{M}$. Namely, it is set

$$
h(t)=v(t), \quad \text { with } t \in \mathbb{R}^{+},
$$

in the equations of the flow dynamics and of the output of $\Sigma_{C}$, where $v(t)$ is assumed to be accessible for measurement.

Hence, the problem of structural disturbance decoupling by feedforward, with reference to the extended linear impulsive system $\Sigma$, is stated as follows.

Problem 2. (Structural disturbance decoupling by feedforward) Let the extended linear impulsive system $\Sigma$ be given. Find a feedforward linear impulsive compensator $\Sigma_{C}$ such that the compensated linear impulsive system

$$
\tilde{\Sigma} \equiv\left\{\begin{array}{l}
\dot{\tilde{x}}(t)=\tilde{A} \tilde{x}(t)+\tilde{H} v(t), \quad \text { with } t \in \mathbb{R}^{+} \backslash \mathcal{T}, \\
\tilde{x}(t)=\tilde{J} \tilde{x}\left(t^{-}\right), \quad \text { with } t=t_{k}, \quad t_{k} \in \mathcal{T}, \\
y(t)=\tilde{C} \tilde{x}(t), \quad \text { with } t \in \mathbb{R}^{+},
\end{array}\right.
$$

where

$$
\begin{aligned}
\tilde{A} & =\left[\begin{array}{cc}
A & B C_{C} \\
0 & A_{C}
\end{array}\right], \\
\tilde{H} & =\left[\begin{array}{c}
B D_{C}+H \\
B_{C}
\end{array}\right], \\
\tilde{J} & =\left[\begin{array}{cc}
J & 0 \\
0 & J_{C}
\end{array}\right], \\
\tilde{C} & =\left[\begin{array}{ll}
C & 0
\end{array}\right],
\end{aligned}
$$

satisfies the requirement that the output $y(t)$ is zero, for all $t \in \mathbb{R}^{+}$, when the initial state is zero, for all the admissible input functions $v(t)$, with $t \in \mathbb{R}^{+}$, and for all the admissible sequences of jump times $\mathcal{T} \in \mathscr{T}$.

The block diagram in Fig. 2 shows the system interconnection taken into consideration in Problem 2 


\section{Structural notions of the geometric approach for linear impulsive systems with nonuniformly spaced state jumps}

The broad literature on disturbance decoupling shows that one of the most powerful tools for handling this control problem is the geometric approach (Wonham, 1985; Basile and Marro, 1992). Indeed, the fundamental notions originally set forth to solve the disturbance decoupling problem for linear time-invariant systems have progressively been extended and adjusted in order to tackle more general problem formulations, addressing various classes of dynamical systems, such as, for instance, systems over rings (Conte and Perdon, 1995), linear parameter varying systems (Conte et al., 2015b), switched linear systems in the case of inaccessible disturbances (Zattoni and Marro, 2013a; Zattoni et al., $2014 \mathrm{a}$; 2016) as well as in the case of combined, measurable and inaccessible, disturbances (Zattoni, 2016a).

Concerning linear impulsive systems (either with unequally spaced or with periodic state jumps), some fundamental notions of the geometric approach, like those of invariance and controlled invariance, have been generalized so as to fit these classes of dynamical systems in some previous papers which dealt with different control problems. To be more specific, these problems were that of output regulation in the presence of periodic state jumps (Zattoni et al., 2015; 2017b) and in the presence of unequally spaced state jumps (Zattoni et al., 2017a), as well as that of inaccessible disturbance decoupling both in the presence of periodic state jumps (Conte et al., 2015a) and nonuniformly spaced state jumps (Perdon et al., 2016b). Hence, the purpose of this section is to collect and review such, recently introduced, structural notions, needed to successfully manage the problem of disturbance decoupling in linear impulsive systems with nonuniformly spaced state jumps, in particular.

For ease of reference, the definitions and the properties of the structural concepts of invariant subspace and of controlled invariant subspace for a linear impulsive system with nonuniformly spaced state jumps are set forth with regard to the extended linear impulsive system previously defined, namely, the system $\Sigma$. However, it is understood that only the macroscopic structure of $\Sigma$ plays a role in the presentation of these notions. In other words, the special structure, shown in (4)-(6), of the matrices of $\Sigma$, deriving from the definition of $\Sigma$ as the output-difference connection between the linear impulsive plant $\Sigma_{P}$ and the modified linear impulsive model $\tilde{\Sigma}_{M}$, is uninfluential to this specific purpose.

The symbols $\mathcal{B}$ and $\mathcal{H}$ are used henceforth to denote the subspaces $\operatorname{Im} B$ and $\operatorname{Im} H$, respectively. Moreover, from now on, the symbol $\mathcal{C}$ stands for the subspace $\operatorname{Ker} C$. Hence, the following definitions and properties, first presented by Zattoni et al. (2015), can be stated.

Definition 1. A subspace $\mathcal{V} \subseteq \mathcal{X}$ is said to be an invariant subspace for the linear impulsive system $\Sigma$ if

$$
\begin{aligned}
& A \mathcal{V} \subseteq \mathcal{V}, \\
& J \mathcal{V} \subseteq \mathcal{V} .
\end{aligned}
$$

A subspace $\mathcal{V}$ satisfying (11), (12) is called an $\mathscr{H}$-invariant subspace.

Definition 2. A subspace $\mathcal{V} \subseteq \mathcal{X}$ is said to be a controlled invariant subspace for the linear impulsive system $\Sigma$ if (12) and

$$
A \mathcal{V} \subseteq \mathcal{V}+\mathcal{B}
$$

hold. A subspace $\mathcal{V}$ satisfying (12), (13) is called an $\mathscr{H}$-controlled invariant subspace.

Proposition 1. A subspace $\mathcal{V} \subseteq \mathcal{X}$, with a basis matrix $V$, is a controlled invariant subspace for the linear impulsive system $\Sigma$ if and only if there exist matrices $L_{A}, L_{J}$, and $M$ such that

$$
\begin{aligned}
A V & =V L_{A}+B M, \\
J V & =V L_{J} .
\end{aligned}
$$

Proposition 2. A subspace $\mathcal{V} \subseteq \mathcal{X}$ is a controlled invariant subspace for the linear impulsive system $\Sigma$ if and only if there exists a linear map $F: \mathcal{X} \rightarrow \mathcal{U}$ such that

$$
(A+B F) \mathcal{V} \subseteq \mathcal{V}
$$

holds along with (12).

Definition 3. Let $\mathcal{V} \subseteq \mathcal{X}$ be a controlled invariant subspace for the linear impulsive system $\Sigma$. Any linear map $F: \mathcal{X} \rightarrow \mathcal{U}$ such that (16) holds is said to be a friend of $\mathcal{V}$.

Proposition 3. The set of all controlled invariant subspaces for the linear impulsive system $\Sigma$ contained in a given subspace $\mathcal{K} \subseteq \mathcal{X}$ is an upper semilattice, henceforth denoted by $\Lambda(\mathcal{K})$, with respect to the sum and the inclusion of subspaces.

Definition 4. The maximum of the upper semilattice of all controlled invariant subspaces for the linear impulsive system $\Sigma$ contained in a given subspace $\mathcal{K} \subseteq \mathcal{X}$ is called the maximal $\mathscr{H}$-controlled invariant subspace contained in $\mathcal{K}$ and is denoted by $\mathcal{V}_{\mathscr{H}}^{*}(\mathcal{K})$.

The subspace $\mathcal{V}_{\mathscr{H}}^{*}(\mathcal{K})$ can be computed through the following algorithm, which was first presented by Conte et al. (2015a).

Algorithm 1. The maximal controlled invariant subspace for the linear impulsive system $\Sigma$ contained in a given 
subspace $\mathcal{K} \subseteq \mathcal{X}$-i.e., the subspace $\mathcal{V}_{\mathscr{H}}^{*}(\mathcal{K})$-is the last term of the nonincreasing sequence of subspaces

$$
\begin{aligned}
& \mathcal{V}_{0}=\mathcal{K}, \\
& \mathcal{V}_{i}=\mathcal{V}_{i-1} \cap A^{-1}\left(\mathcal{V}_{i}+\mathcal{B}\right) \cap J^{-1} \mathcal{V}_{i}, \\
& i=1, \ldots, k
\end{aligned}
$$

where $k \leq n-1$ is the least integer such that $\mathcal{V}_{k+1}=\mathcal{V}_{k}$.

It is worth pointing out that, in the remainder of this work, the only upper semilattice of controlled invariant subspaces for the linear impulsive system $\Sigma$ playing a role in connection with the solution of the structural disturbance decoupling problem is the upper semilattice of the controlled invariant subspaces contained in the subspace $\mathcal{C}$ (i.e., the null space of the output distribution matrix $C$ of $\Sigma$ ). For this reason, the corresponding maximal element, which is $\mathcal{V}_{\mathscr{H}}^{*}(\mathcal{C})$, will be briefly denoted by $\mathcal{V}_{\mathscr{H}}^{*}$ hereafter. In other words, the $\operatorname{argument} \mathcal{C}$ will henceforth be dropped since no confusion may arise.

\section{Problem solution: Structural disturbance decoupling by feedforward}

The purpose of this section is to demonstrate a necessary and sufficient condition for the existence of a solution to the problem of structural disturbance decoupling by feedforward for linear impulsive systems subject to unequally spaced state jumps. As will be shown in the following, a necessary and sufficient condition for solvability of Problem 2 (formulated in Section 3) can be expressed in geometric terms by exploiting the concepts introduced in Section 4

It is worth premising that the necessary and sufficient solvability condition that will be the main result of this section (Theorem 1) consists in a subspace inclusion and, as such, it expresses a concept which is independent of coordinates. However, since the proof of sufficiency is constructive-i.e., it hinges on the design of a suitable feedforward linear impulsive compensator-it is convenient to provide some preliminary results, aimed at expressing that condition in a coordinate-dependent form, with respect to a conveniently chosen basis of the state space of the given linear impulsive system. Indeed, this is the purpose of Lemmas 1 and 2 in the following.

As to the proof of necessity, this is based on the notion of unobservable subspace of a linear impulsive system with nonuniformly spaced state jumps. Hence, also the definition and an algorithmic characterization of this geometric object will be given before the statement of the solvability condition. To be more specific, Definition 5 in the following represents the formal definition of the unobservable subspace adapted to the case of linear impulsive systems with nonuniformly spaced state jumps. Then, Lemma 3 states a property of the unobservable subspace which suggests an algorithmic procedure to compute it. For ease of reference, both the definition and the characterizing property will be formulated addressing the compensated linear impulsive system $\tilde{\Sigma}$.

Lemma 1. Let the extended linear impulsive system $\Sigma$ be given. Let $\mathcal{V}_{\mathscr{H}}^{*}$ be the maximal controlled invariant subspace for $\Sigma$ contained in $\mathcal{C}$. Let $F: \mathcal{X} \rightarrow \mathcal{U}$ be a friend of $\mathcal{V}_{\mathscr{H}}^{*}$. Perform the state space basis transformation $T$, defined by $T=\left[T_{1} T_{2}\right]$, where $T_{1}$ is a basis matrix of $\mathcal{V}_{\mathscr{H}}^{*}$ and $T_{2}$ is any matrix such that $T$ is square and nonsingular. Then, with respect to the new coordinates, the matrices $A^{\prime}+B^{\prime} F^{\prime}=T^{-1}(A+B F) T$ and $J^{\prime}=T^{-1} J T$, partitioned according to $T$, have the characteristic upper block-triangular structure shown below:

$$
\begin{aligned}
& A^{\prime}+B^{\prime} F^{\prime}=T^{-1}(A+B F) T \\
& \quad=\left[\begin{array}{cc}
A_{11}^{\prime}+B_{1}^{\prime} F_{1}^{\prime} & A_{12}^{\prime}+B_{1}^{\prime} F_{2}^{\prime} \\
0 & A_{22}^{\prime}+B_{2}^{\prime} F_{2}^{\prime}
\end{array}\right], \\
& J^{\prime}=T^{-1} J T=\left[\begin{array}{cc}
J_{11}^{\prime} & J_{12}^{\prime} \\
0 & J_{22}^{\prime}
\end{array}\right] .
\end{aligned}
$$

Moreover, the matrix $C^{\prime}=C T$, also partitioned consistently with $T$, has the structure

$$
C^{\prime}=C T=\left[\begin{array}{ll}
0 & C_{2}^{\prime}
\end{array}\right]
$$

Proof. Concerning the matrix $A^{\prime}+B^{\prime} F^{\prime}$, the structural zero submatrix at the lower left corner or, equivalently, the condition

$$
A_{21}^{\prime}+B_{2}^{\prime} F_{1}^{\prime}=0
$$

is due to $(A+B F)$-invariance of $\mathcal{V}_{\mathscr{H}}^{*}$. Likewise, the structural zero submatrix at the lower left corner of $J^{\prime}$ is due to $J$-invariance of $\mathcal{V}_{\mathscr{H}}^{*}$. Furthermore, the structural zero submatrix in $C^{\prime}$ ensues from $\mathcal{V}_{\mathscr{H}}^{*} \subseteq \mathcal{C}$.

Lemma 2. Let the extended linear impulsive system $\Sigma$ be given. Let $\mathcal{V}_{\mathscr{H}}^{*}$ be the maximal controlled invariant subspace for $\Sigma$ contained in $\mathcal{C}$. Let $\mathcal{H}$ be the subspace spanned by the column vectors of the disturbance input distribution matrix $H$. Let the subspace $\mathcal{P} \subseteq \mathcal{V}_{\mathscr{H}}^{*}$ be such that

$$
\begin{aligned}
\mathcal{B} \cap \mathcal{P} & =\{0\}, \\
\left(\mathcal{B} \cap \mathcal{V}_{\mathscr{H}}^{*}\right)+\mathcal{P} & =\mathcal{V}_{\mathscr{H}}^{*} .
\end{aligned}
$$

Let $r$ be the dimension of $\mathcal{P}$ and let $P \in \mathbb{R}^{n \times r}$ be a matrix whose column vectors form a basis of $\mathcal{P}$. Perform the state space basis transformation $T$, defined by $T=\left[\begin{array}{ll}T_{1} & T_{2}\end{array}\right]$, where $T_{1}$ is a basis matrix of $\mathcal{V}_{\mathscr{H}}^{*}$ and $T_{2}$ is any matrix such that $T$ is square and nonsingular. Then, with respect to the new coordinates, the matrix $P^{\prime}=T^{-1} P$, partitioned according to $T$, has the structure

$$
P^{\prime}=T^{-1} P=\left[\begin{array}{c}
P_{1}^{\prime} \\
0
\end{array}\right] .
$$


Moreover, the subspace inclusion

$$
\mathcal{H} \subseteq \mathcal{B}+\mathcal{V}_{\mathscr{H}}^{*}
$$

holds if and only if there exists a unique pair of matrices $\Phi \in \mathbb{R}^{p \times m}$ and $\Psi \in \mathbb{R}^{r \times m}$ such that

$$
H^{\prime}=B^{\prime} \Phi+P^{\prime} \Psi,
$$

where $H^{\prime}=T^{-1} H, \quad B^{\prime}=T^{-1} B$ and $P^{\prime}=T^{-1} P$. Equivalently, with reference to the partition of $H^{\prime}, B^{\prime}$, and $P^{\prime}$ consistent with the partition of $T$, the subspace inclusion (26) holds if and only if there exists a unique pair of matrices $\Phi \in \mathbb{R}^{p \times m}$ and $\Psi \in \mathbb{R}^{r \times m}$ such that

$$
\left[\begin{array}{l}
H_{1}^{\prime} \\
H_{2}^{\prime}
\end{array}\right]=\left[\begin{array}{l}
B_{1}^{\prime} \\
B_{2}^{\prime}
\end{array}\right] \Phi+\left[\begin{array}{c}
P_{1}^{\prime} \\
0
\end{array}\right] \Psi
$$

Proof. First, note that the structural zero submatrix in $P^{\prime}$ shown in 25) is due to $\mathcal{P} \subseteq \mathcal{V}_{\mathscr{H}}^{*}$. Secondly, note that 23) implies

$$
\left(\mathcal{B} \cap \mathcal{V}_{\mathscr{H}}^{*}\right) \cap \mathcal{P}=\{0\} .
$$

Hence, (24) and (29) are equivalent to

$$
\mathcal{V}_{\mathscr{H}}^{*}=\left(\mathcal{V}_{\mathscr{H}}^{*} \cap \mathcal{B}\right) \oplus \mathcal{P} \text {. }
$$

By summing $\mathcal{B}$ to both members of (30) and taking into account that $\mathcal{B} \supseteq\left(\mathcal{V}_{\mathscr{H}}^{*} \cap \mathcal{B}\right)$ and that $(23)$ holds, one gets

$$
\mathcal{B}+\mathcal{V}_{\mathscr{H}}^{*}=\mathcal{B} \oplus \mathcal{P}
$$

In light of 31, the inclusion 26) is equivalent to

$$
\mathcal{H} \subseteq \mathcal{B} \oplus \mathcal{P}
$$

In particular, the direct sum in (32) is equivalent to the existence of a unique pair of matrices $\Phi$ and $\Psi$, of appropriate dimensions, such that 27) or, equivalently, (28) hold. In fact, each column vector of $H^{\prime}$, which is a basis matrix of $\mathcal{H}$ with respect to the new coordinates, can be expressed in a unique way as a linear combination of the column vectors of $B^{\prime}$ and $P^{\prime}$, which are basis matrices of $\mathcal{B}$ and $\mathcal{P}$, respectively, in the new coordinates.

In order to introduce the notion of unobservable subspace of a linear impulsive system subject to nonuniformly spaced state jumps, it is worth first reviewing the concept of unobservable subspace for the sole flow dynamics. The flow dynamics is a linear time-invariant dynamics, and the output equation is the same for both the flow and the jump dynamics. Therefore, the unobservable subspace of the flow dynamics - which is the set of the initial states that give rise to free state trajectories corresponding to zero output-coincides with the subspace which enjoys the property of being the maximal invariant subspace, with respect to the linear map defining the system dynamics, contained in the null space of the output distribution matrix. As mentioned at the beginning of this section, the statements concerning the unobservable subspace will be presented with reference to the compensated linear impulsive system $\tilde{\Sigma}$. Consequently, the unobservable subspace of the flow dynamics of $\tilde{\Sigma}$-henceforth denoted by $\tilde{\mathcal{O}}$ - can be characterized as $\max \mathcal{J}(\tilde{A}, \tilde{\mathcal{C}})$, where $\tilde{\mathcal{C}}$ denotes the kernel of the output distribution matrix $\tilde{C}$, i.e., $\tilde{\mathcal{C}}=\operatorname{Ker} \tilde{C}$. Hence, the definition of the unobservable subspace of $\tilde{\Sigma}$ and its characterization can be stated as follows.

Definition 5. The set of all the initial states of the linear impulsive system with nonuniformly spaced state jumps $\tilde{\Sigma}$ giving rise to state motions corresponding to zero output for all the admissible sequences of jump times $\mathcal{T} \in \mathscr{T}$ is called the unobservable subspace of $\tilde{\Sigma}$ and is denoted by $\tilde{\mathcal{Q}}$.

Lemma 3. The unobservable subspace $\tilde{\mathcal{Q}}$ of the linear impulsive system with nonuniformly spaced state jumps $\tilde{\Sigma}$ is the maximal invariant subspace of $\tilde{\Sigma}$ contained in the unobservable subspace $\tilde{\mathcal{O}}$ of the flow dynamics of $\tilde{\Sigma}$.

Proof. First, note that the unobservable subspace $\tilde{\mathcal{Q}}$ is a locus of trajectories of the linear impulsive system with nonuniformly spaced state jumps $\tilde{\Sigma}$, contained in the kernel $\tilde{\mathcal{C}}$ of the output distribution matrix $\tilde{C}$. As a locus of trajectories of the linear impulsive dynamics, $\tilde{\mathcal{Q}}$ is invariant under the linear map $\tilde{J} e^{\tilde{A} t}$ for any given $t \in \mathbb{R}^{+}$, namely,

$$
\tilde{J} e^{\tilde{A} t} \tilde{\mathcal{Q}} \subseteq \tilde{\mathcal{Q}},
$$

for any given $t \in \mathbb{R}^{+}$. Secondly, observe that $\tilde{\mathcal{Q}}$ is a locus of trajectories of the flow dynamics of $\tilde{\Sigma}$ contained in $\tilde{\mathcal{C}}$. As a locus of trajectories of the flow dynamics, $\tilde{\mathcal{Q}}$ is invariant under the linear map $\tilde{A}$, i.e.,

$$
\tilde{A} \tilde{\mathcal{Q}} \subseteq \tilde{\mathcal{Q}} .
$$

By applying the state space basis transformation

$$
\tilde{T}=\left[\begin{array}{ll}
\tilde{T}_{1} & \tilde{T}_{2}
\end{array}\right]
$$

where $\tilde{T}_{1}$ is a matrix whose column vectors form a basis of $\tilde{\mathcal{Q}}$ and $\tilde{T}_{2}$ is any matrix such that $\tilde{T}$ is square and nonsingular, one obtains

$$
\begin{aligned}
& \tilde{A}^{\prime}=\tilde{T}^{-1} \tilde{A} \tilde{T}=\left[\begin{array}{cc}
\tilde{A}_{11}^{\prime} & \tilde{A}_{12}^{\prime} \\
0 & \tilde{A}_{22}^{\prime}
\end{array}\right], \\
& \tilde{J}^{\prime}=\tilde{T}^{-1} \tilde{J} \tilde{T}=\left[\begin{array}{cc}
\tilde{J}_{11}^{\prime} & \tilde{J}_{12}^{\prime} \\
\tilde{J}_{21}^{\prime} & \tilde{J}_{22}^{\prime}
\end{array}\right],
\end{aligned}
$$

where the structural zero submatrix at the lower left corner of $\tilde{A}^{\prime}$, shown in (35), is due to (34). By taking into account (35) and (36), one gets

$$
\tilde{J}^{\prime} e^{\tilde{A}^{\prime} t}=\left[\begin{array}{cc}
\tilde{J}_{11}^{\prime} e^{\tilde{A}_{11}^{\prime} t} & * \\
\tilde{J}_{21}^{\prime} e^{\tilde{A}_{11}^{\prime} t} & *
\end{array}\right],
$$


for any given $t \in \mathbb{R}^{+}$, where the symbol ' $*$ ' denotes a matrix block whose expression is of no special interest to this discussion and, therefore, has not been specified to avoid heavier (inessential) developments. By virtue of (33), from 37) it follows that

$$
\tilde{J}_{21}^{\prime} e^{\tilde{A}_{11}^{\prime} t}=0
$$

for any given $t \in \mathbb{R}^{+}$. Since $e^{\tilde{A}_{11}^{\prime} t}$ is nonzero for all $t \in \mathbb{R}$, 38) holds if and only if $\tilde{J}_{21}^{\prime}=0$, which, in turn, is equivalent to $\tilde{J}$-invariance of $\tilde{\mathcal{Q}}$. Consequently, $\tilde{\mathcal{Q}}$, being both $\tilde{A}$-invariant and $\tilde{J}$-invariant, is invariant for the linear impulsive system $\tilde{\Sigma}$. Moreover, $\tilde{\mathcal{Q}}$, as an invariant subspace for the linear impulsive system $\tilde{\Sigma}$ contained in $\tilde{\mathcal{C}}$, is contained in $\tilde{\mathcal{O}}$, since the latter is the maximal invariant subspace of $\tilde{\Sigma}$ contained in $\tilde{\mathcal{C}}$. Finally, maximality of $\tilde{\mathcal{Q}}$ can be easily shown by contradiction.

As mentioned above, the previous lemmas pave the way to the proof of the necessary and sufficient condition for solvability Problem 2 , which can be stated as follows.

Theorem 1. Let the linear impulsive system with nonuniformly spaced state jumps $\Sigma$ be given. Let $\mathcal{V}_{\mathscr{H}}^{*}$ be the maximal controlled invariant subspace of $\Sigma$ contained in $\mathcal{C}$. Let $\mathcal{B}$ and $\mathcal{H}$ be the subspaces respectively spanned by the column vectors of the control input distribution matrix $B$ and by the column vectors of the disturbance input distribution matrix H. Problem 2 has a solution if and only if (26) holds.

Proof.

(Sufficiency) First, note that this part of the proof will be entirely developed by referring to the coordinates considered in Lemmas 11 and 2, Let (26) hold. Let the linear map $F: \mathcal{X} \rightarrow \mathcal{U}$ be a friend of $\mathcal{V}_{\mathscr{H}}^{*}$. Hence, with respect to the coordinates introduced in Lemmas 1 and 2. (19), 20), 21), and 28) hold. With respect to the same coordinates, let the feedforward linear impulsive compensator $\Sigma_{C}$ be defined by the following matrices:

$$
\begin{aligned}
A_{C}^{\prime} & =A_{11}^{\prime}+B_{1}^{\prime} F_{1}^{\prime}, \\
B_{C}^{\prime} & =P_{1}^{\prime} \Psi, \\
J_{C}^{\prime} & =J_{11}^{\prime}, \\
C_{C}^{\prime} & =F_{1}^{\prime}, \\
D_{C}^{\prime} & =-\Phi .
\end{aligned}
$$

Then, it will be shown that the compensator $\Sigma_{C}$ thus defined, with zero initial state, solves Problem 2

To this aim, recall that the cascade of the feedforward linear impulsive compensator $\Sigma_{C}$ with the extended linear impulsive system $\Sigma$, denoted by $\tilde{\Sigma}$ in Problem 2 is described by the matrices shown in (7)-(10). Consequently, with respect to new coordinates introduced in Lemmas 1 and 2, and taking into account (39)-43), it ensues that $\tilde{\Sigma}$ is described by (44). In particular, observe that the state space basis transformation $T$, introduced in Lemmas 1 and 2. has been applied to the original state $x(t)$, so that the new state $z(t)=T^{-1} x(t)$, partitioned according to $T$, is given by

$$
z(t)=\left[\begin{array}{l}
z_{1}(t) \\
z_{2}(t)
\end{array}\right], \quad \text { with } t \in \mathbb{R}^{+} .
$$

Moreover, the state of the feedforward linear impulsive compensator with respect to these coordinates has been denoted by $z_{C}(t)$. Also observe that (28) has been taken into account in writing (44). Next, by applying the change of variables

$$
\zeta(t)=z_{1}(t)-z_{C}(t), \quad \text { with } t \in \mathbb{R}^{+},
$$

the system $\tilde{\Sigma}$ can be written as (45), where (22) has been taken into account. Hence, the assumption of zero initial state implies $\zeta(t)=0$ and $z_{2}(t)=0$, for all $t \in \mathbb{R}^{+}$, which, in particular, also implies $y(t)=0$, for all $t \in \mathbb{R}^{+}$, for all the admissible input functions $v(t)$, with $t \in \mathbb{R}^{+}$, and for all the admissible sequences of jump times $\mathcal{T} \in \mathscr{T}$.

(Necessity) Let Problem 2 have a solution. Then, there exists a feedforward linear impulsive compensator $\Sigma_{C}$ such that the compensated linear impulsive system $\tilde{\Sigma}$ satisfies the condition that the subspace $\tilde{\mathcal{H}}$, spanned by the column vectors of $\tilde{H}$, is contained in the unobservable subspace $\tilde{\mathcal{Q}}$. Refer to the coordinates introduced in Lemmas 1 and 2 Hence, the compensated linear impulsive system $\tilde{\Sigma}$ is described by the following matrices:

$$
\begin{aligned}
\tilde{A}^{\prime}= & {\left[\begin{array}{ccc}
A_{11}^{\prime} & A_{12}^{\prime} & B_{1}^{\prime} C_{C}^{\prime} \\
A_{21}^{\prime} & A_{22}^{\prime} & B_{2}^{\prime} C_{C}^{\prime} \\
0 & 0 & A_{C}^{\prime}
\end{array}\right], } \\
\tilde{J}^{\prime}= & {\left[\begin{array}{ccc}
J_{11}^{\prime} & J_{12}^{\prime} & 0 \\
0 & J_{22}^{\prime} & 0 \\
0 & 0 & J_{C}^{\prime}
\end{array}\right], } \\
\tilde{C}^{\prime}= & {\left[\begin{array}{ccc}
0 & C_{2}^{\prime} & 0
\end{array}\right], } \\
\tilde{H}^{\prime}= & {\left[\begin{array}{c}
B_{1}^{\prime} D_{C}^{\prime}+H_{1}^{\prime} \\
B_{2}^{\prime} D_{C}^{\prime}+H_{2}^{\prime} \\
B_{C}^{\prime}
\end{array}\right] . }
\end{aligned}
$$

Let $\tilde{Q}^{\prime}$ be a full rank matrix whose column vectors span the unobservable subspace $\tilde{\mathcal{Q}}$ of $\tilde{\Sigma}$. According to the partition shown in (46)-49, let $\tilde{Q}^{\prime}$ be partitioned as

$$
\tilde{Q}^{\prime}=\left[\begin{array}{l}
Q_{1}^{\prime} \\
Q_{2}^{\prime} \\
Q_{C}^{\prime}
\end{array}\right]
$$

Hence, it will be shown that the subspace $\mathcal{V} \subseteq \mathcal{X}$, defined by

$$
\mathcal{V}=\operatorname{Im} V^{\prime}=\operatorname{Im}\left[\begin{array}{l}
Q_{1}^{\prime} \\
Q_{2}^{\prime}
\end{array}\right]
$$




$$
\tilde{\Sigma} \equiv\left\{\begin{aligned}
\dot{z}_{1}(t) & =A_{11}^{\prime} z_{1}(t)+A_{12}^{\prime} z_{2}(t)+B_{1}^{\prime} F_{1}^{\prime} z_{C}(t)+P_{1}^{\prime} \Psi v(t), \quad \text { with } t \in \mathbb{R}^{+} \backslash \mathcal{T}, \\
\dot{z}_{2}(t) & =A_{21}^{\prime} z_{1}(t)+A_{22}^{\prime} z_{2}(t)+B_{2}^{\prime} F_{1}^{\prime} z_{C}(t), \quad \text { with } t \in \mathbb{R}^{+} \backslash \mathcal{T}, \\
\dot{z}_{C}(t) & =\left(A_{11}^{\prime}+B_{1}^{\prime} F_{1}^{\prime}\right) z_{C}(t)+P_{1}^{\prime} \Psi v(t), \quad \text { with } t \in \mathbb{R}^{+} \backslash \mathcal{T} \\
z_{1}(t) & =J_{11}^{\prime} z_{1}\left(t^{-}\right)+J_{12}^{\prime} z_{2}\left(t^{-}\right), \quad \text { with } t=t_{k}, t_{k} \in \mathcal{T}, \\
z_{2}(t) & =J_{22}^{\prime} z_{2}\left(t^{-}\right), \quad \text { with } t=t_{k}, \quad t_{k} \in \mathcal{T}, \\
z_{C}(t) & =J_{11}^{\prime} z_{C}\left(t^{-}\right), \quad \text { with } t=t_{k}, t_{k} \in \mathcal{T}, \\
y(t) & =C_{2}^{\prime} z_{2}(t), \quad \text { with } t \in \mathbb{R}^{+} .
\end{aligned}\right.
$$

$$
\tilde{\Sigma}_{\tilde{\Sigma}} \equiv\left\{\begin{aligned}
\dot{\zeta}(t) & =A_{11}^{\prime} \zeta(t)+A_{12}^{\prime} z_{2}(t), \quad \text { with } t \in \mathbb{R}^{+} \backslash \mathcal{T}, \\
\dot{z}_{2}(t) & =A_{21}^{\prime} \zeta(t)+A_{22}^{\prime} z_{2}(t), \quad \text { with } t \in \mathbb{R}^{+} \backslash \mathcal{T}, \\
\dot{z}_{C}(t) & =\left(A_{11}^{\prime}+B_{1}^{\prime} F_{1}^{\prime}\right) z_{C}(t)+P_{1}^{\prime} \Psi v(t), \quad \text { with } t \in \mathbb{R}^{+} \backslash \mathcal{T}, \\
\zeta(t) & =J_{11}^{\prime} \zeta\left(t^{-}\right)+J_{12}^{\prime} z_{2}\left(t^{-}\right), \quad \text { with } t=t_{k}, \quad t_{k} \in \mathcal{T} \\
z_{2}(t) & =J_{22}^{\prime} z_{2}\left(t^{-}\right), \quad \text { with } t=t_{k}, \quad t_{k} \in \mathcal{T}, \\
z_{C}(t) & =J_{11}^{\prime} z_{C}\left(t^{-}\right), \quad \text { with } t=t_{k}, t_{k} \in \mathcal{T}, \\
y(t) & =C_{2}^{\prime} z_{2}(t), \quad \text { with } t \in \mathbb{R}^{+} .
\end{aligned}\right.
$$

is a controlled invariant subspace for the extended linear impulsive system $\Sigma$, enjoying the property of being contained in $\mathcal{C}$ and also satisfying the condition

$$
\mathcal{H} \subseteq \mathcal{B}+\mathcal{V} .
$$

To this aim, first note that $\tilde{A}$-invariance of $\tilde{\mathcal{Q}}$ is equivalent to the existence of a matrix $L_{A}$ such that

$$
\tilde{A}^{\prime} \tilde{Q}^{\prime}=\tilde{Q}^{\prime} L_{A}
$$

or, equivalently,

$$
\left[\begin{array}{ccc}
A_{11}^{\prime} & A_{12}^{\prime} & B_{1}^{\prime} C_{C}^{\prime} \\
A_{21}^{\prime} & A_{22}^{\prime} & B_{2}^{\prime} C_{C}^{\prime} \\
0 & 0 & A_{C}^{\prime}
\end{array}\right]\left[\begin{array}{l}
Q_{1}^{\prime} \\
Q_{2}^{\prime} \\
Q_{C}^{\prime}
\end{array}\right]=\left[\begin{array}{l}
Q_{1}^{\prime} \\
Q_{2}^{\prime} \\
Q_{C}^{\prime}
\end{array}\right] L_{A}
$$

hold. The first two blocks of rows in (54) can also be written as

$$
\begin{aligned}
& {\left[\begin{array}{cc}
A_{11}^{\prime} & A_{12}^{\prime} \\
A_{21}^{\prime} & A_{22}^{\prime}
\end{array}\right]\left[\begin{array}{l}
Q_{1}^{\prime} \\
Q_{2}^{\prime}
\end{array}\right]} \\
& \quad=\left[\begin{array}{l}
Q_{1}^{\prime} \\
Q_{2}^{\prime}
\end{array}\right] L_{A}-\left[\begin{array}{l}
B_{1}^{\prime} \\
B_{2}^{\prime}
\end{array}\right] C_{C}^{\prime} Q_{C}^{\prime} .
\end{aligned}
$$

Let the matrix $M$ be defined as $M=-C_{C}^{\prime} Q_{C}^{\prime}$. Hence, (55) shows that there exists a pair of matrices $L_{A}$ and $M$ such that

$$
A^{\prime} V^{\prime}=V^{\prime} L_{A}+B^{\prime} M
$$

holds, which is equivalent to stating that $\mathcal{V}$, defined by (51), is an $(A, \mathcal{B})$-controlled invariant subspace. Similarly, $\tilde{J}$-invariance of $\tilde{\mathcal{Q}}$ is equivalent to the existence of a matrix $L_{J}$ such that

$$
\tilde{J}^{\prime} \tilde{Q}^{\prime}=\tilde{Q}^{\prime} L_{J}
$$

or, equivalently,

$$
\left[\begin{array}{ccc}
J_{11}^{\prime} & J_{12}^{\prime} & 0 \\
0 & J_{22}^{\prime} & 0 \\
0 & 0 & J_{C}^{\prime}
\end{array}\right]\left[\begin{array}{l}
Q_{1}^{\prime} \\
Q_{2}^{\prime} \\
Q_{C}^{\prime}
\end{array}\right]=\left[\begin{array}{l}
Q_{1}^{\prime} \\
Q_{2}^{\prime} \\
Q_{C}^{\prime}
\end{array}\right] L_{J}
$$

hold. The first two blocks of rows in (58) can also be written as

$$
\left[\begin{array}{cc}
J_{11}^{\prime} & J_{12}^{\prime} \\
0 & J_{22}^{\prime}
\end{array}\right]\left[\begin{array}{l}
Q_{1}^{\prime} \\
Q_{2}^{\prime}
\end{array}\right]=\left[\begin{array}{l}
Q_{1}^{\prime} \\
Q_{2}^{\prime}
\end{array}\right] L_{J} .
$$

Hence, (59) shows that there exists a matrix $L_{J}$ such that

$$
J^{\prime} V^{\prime}=V^{\prime} L_{J}
$$

holds, which is equivalent to stating that $\mathcal{V}$, defined by (51), is a $J$-invariant subspace. Hence, (56) and (60) show that $\mathcal{V}$ is a controlled invariant subspace of the extended linear impulsive system $\Sigma$. Moreover, the inclusion $\tilde{\mathcal{Q}} \subseteq \tilde{\mathcal{C}}$ is equivalent to $C^{\prime} Q^{\prime}=0$ or

$$
\left[\begin{array}{lll}
0 & C_{2}^{\prime} & 0
\end{array}\right]\left[\begin{array}{l}
Q_{1}^{\prime} \\
Q_{2}^{\prime} \\
Q_{C}^{\prime}
\end{array}\right]=0 .
$$

By considering the product only between the first two, respective, matrix blocks, one gets

$$
\left[\begin{array}{ll}
0 & C_{2}^{\prime}
\end{array}\right]\left[\begin{array}{l}
Q_{1}^{\prime} \\
Q_{2}^{\prime}
\end{array}\right]=0
$$

which is equivalent to the inclusion $\mathcal{V} \subseteq \mathcal{C}$. Furthermore, the inclusion $\tilde{\mathcal{H}} \subseteq \tilde{\mathcal{Q}}$ is equivalent to the existence of a matrix $X$, of suitable dimensions, such that $\tilde{H}^{\prime}=\tilde{Q}^{\prime} X$ or, equivalently,

$$
\left[\begin{array}{c}
B_{1}^{\prime} D_{C}^{\prime}+H_{1}^{\prime} \\
B_{2}^{\prime} D_{C}^{\prime}+H_{2}^{\prime} \\
B_{C}^{\prime}
\end{array}\right]=\left[\begin{array}{c}
Q_{1}^{\prime} \\
Q_{2}^{\prime} \\
Q_{C}^{\prime}
\end{array}\right] X
$$

hold. By only considering the first two blocks of rows in (63), one obtains

$$
\left[\begin{array}{l}
H_{1}^{\prime} \\
H_{2}^{\prime}
\end{array}\right]=-\left[\begin{array}{l}
B_{1}^{\prime} \\
B_{2}^{\prime}
\end{array}\right] D_{C}^{\prime}+\left[\begin{array}{l}
Q_{1}^{\prime} \\
Q_{2}^{\prime}
\end{array}\right] X
$$


or, equivalently,

$$
H^{\prime}=B^{\prime} Y+Q^{\prime} X
$$

where $Y=-D_{C}^{\prime}$. Equation (65) shows that the inclusion

$$
\mathcal{H} \subseteq \mathcal{B}+\mathcal{V}
$$

holds. Note that $\mathcal{V}$, as a controlled invariant invariant subspace of the linear impulsive system $\Sigma$ contained in $\mathcal{C}$, is contained in $\mathcal{V}_{\mathscr{H} \mathcal{C}}^{*}$. Hence, in light of this consideration, (66) implies that (26) holds.

\section{Problem solution: Structural model matching by output feedback}

The objective of this section is to show that the problem of structural disturbance decoupling by feedforward, formulated for the extended linear impulsive system in Section 3 and solved in Section 5, is equivalent to the structural model matching problem by output feedback stated for the original system and model in Section 2. In other words, such equivalence means that a linear impulsive compensator solves any of these problems if and only if it solves the other one. The following theorem provides the formal statement (and the consequent proof) of this result. It is just worth mentioning that, in accordance with the statement above, the output variable is postulated to be zero throughout the proof.

Theorem 2. Let the linear impulsive system $\Sigma_{P}$ and the linear impulsive model $\Sigma_{M}$ be given. A linear impulsive compensator $\Sigma_{C}$ solves Problem 2 if and only if it solves Problem 1 .

Proof.

(Sufficiency) Let the linear impulsive compensator $\Sigma_{C}$ solve Problem 1 Hence, the overall compensated linear impulsive system with measurement feedback-henceforth denoted by $\tilde{\Sigma}^{\prime}$-is described by 67). Note that, since $\Sigma_{C}$ solves Problem 1 on the assumption of zero initial state, the output of $\tilde{\Sigma}^{\prime}$ satisfies the condition that $y(t)=0$, for all $t \in \mathbb{R}^{+}$, for all the admissible input functions $d(t)$, with $t \in \mathbb{R}^{+}$, and for all the admissible sequences of jump times $\mathcal{T} \in \mathscr{T}$. Hence, one can replace $y_{P}(t)=C_{P} x_{P}(t)$ with $y_{M}(t)=C_{M} x_{M}(t)$ in the state equations of $\tilde{\Sigma}^{\prime}$. Thus, the new system, henceforth denoted by $\tilde{\Sigma}^{\prime \prime}$, is described by (68). Moreover, since $y(t)=0$ for all $t \in \mathbb{R}^{+}$, for all the admissible $d(t)$, with $t \in \mathbb{R}^{+}$, this condition holds, in particular, by picking $d(t)=C_{M} x_{M}(t)+v(t)$, where $v(t)$, with $t \in \mathbb{R}^{+}$, denotes any admissible input function. Then, the resulting system is the linear impulsive system $\tilde{\Sigma}$ considered in Problem 2, as shown by (69), derived from those of $\tilde{\Sigma}^{\prime \prime}$ with the replacement mentioned above. The equations of $\tilde{\Sigma}$, which hold with $y(t)=0$ for all $t \in \mathbb{R}^{+}$, for all the admissible $v(t)$, with $t \in \mathbb{R}^{+}$, and for all the admissible sequences of jump times $\mathcal{T} \in \mathscr{T}$, show that the linear impulsive compensator $\Sigma_{C}$ also solves Problem 2, i.e., the problem of decoupling the signal $v(t)$, with $t \in \mathbb{R}^{+}$, in the extended linear impulsive system $\Sigma$, including the modified linear impulsive model $\tilde{\Sigma}_{M}$.

(Necessity) Let the linear impulsive compensator $\Sigma_{C}$ solve Problem 2. Then, in order to show that $\Sigma_{C}$ also solves Problem 1 the same reasoning presented in the proof of sufficiency can be followed backward, i.e., from $\tilde{\Sigma}$ to $\tilde{\Sigma}^{\prime}$.

\section{Conclusions}

In this work, the problem of finding an output feedback linear impulsive compensator which ensures that the forced response of a given linear impulsive system matches that of a given linear impulsive model, for all the admissible inputs and for all the admissible sequences of jump time instants, has been formulated on the general assumption that the jump time instants are not equally spaced in time, but satisfy the constraint that their set has no accumulation points on the time axis. The problem of structural model matching has been tackled by reducing it to an equivalent problem of structural disturbance decoupling by feedforward. Solvability of the structural disturbance decoupling problem has been characterized through a necessary and sufficient condition. Equivalence between the original model matching problem by output feedback and the disturbance decoupling problem by feedforward has been formally established. A computational procedure for the synthesis of the linear impulsive compensator that solves both the equivalent problems has been illustrated through the proof of sufficiency of the solvability condition. In fact, the matrices of the compensator are constructed on the basis of the results set forth in the preceding lemmas.

\section{Acknowledgment}

The author wishes to thank the anonymous reviewers for their constructive comments.

\section{References}

Basile, G. and Marro, G. (1992). Controlled and Conditioned Invariants in Linear System Theory, Prentice Hall, Englewood Cliffs, NJ.

Carnevale, D., Galeani, S., Menini, L. and Sassano, M. (2016). Hybrid output regulation for linear systems with periodic jumps: Solvability conditions, structural implications and semi-classical solutions, IEEE Transactions on Automatic Control 61(9): 2416-2431.

Carnevale, D., Galeani, S. and Sassano, M. (2014a). Francis equations vs invariant subspace algorithm for hybrid output 


$$
\tilde{\Sigma}^{\prime} \equiv\left\{\begin{aligned}
\dot{x}_{P}(t) & =\left(A_{P}-B_{P} D_{C} C_{P}\right) x_{P}(t)+B_{P} C_{C} x_{C}(t)+B_{P} D_{C} d(t), \quad \text { with } t \in \mathbb{R}^{+} \backslash \mathcal{T}, \\
\dot{x}_{M}(t) & =A_{M} x_{M}(t)+B_{M} d(t), \quad \text { with } t \in \mathbb{R}^{+} \backslash \mathcal{T}, \\
\dot{x}_{C}(t) & =-B_{C} C_{P} x_{P}(t)+A_{C} x_{C}(t)+B_{C} d(t), \quad \text { with } t \in \mathbb{R}^{+} \backslash \mathcal{T}, \\
x_{P}(t) & =J_{P} x_{P}\left(t^{-}\right), \quad \text { with } t=t_{k}, t_{k} \in \mathcal{T}, \\
x_{M}(t) & =J_{M} x_{M}\left(t^{-}\right), \quad \text { with } t=t_{k}, t_{k} \in \mathcal{T}, \\
x_{C}(t) & =J_{C} x_{C}\left(t^{-}\right), \quad \text { with } t=t_{k}, t_{k} \in \mathcal{T} \\
y(t) & =C_{P} x_{P}(t)-C_{M} x_{M}(t), \text { with } t \in \mathbb{R}^{+} .
\end{aligned}\right.
$$

$$
\tilde{\Sigma}^{\prime \prime} \equiv\left\{\begin{aligned}
\dot{x}_{P}(t) & =A_{P} x_{P}(t)-B_{P} D_{C} C_{M} x_{M}(t)+B_{P} C_{C} x_{C}(t)+B_{P} D_{C} d(t), \quad \text { with } t \in \mathbb{R}^{+} \backslash \mathcal{T}, \\
\dot{x}_{M}(t) & =A_{M} x_{M}(t)+B_{M} d(t), \quad \text { with } t \in \mathbb{R}^{+} \backslash \mathcal{T}, \\
\dot{x}_{C}(t) & =-B_{C} C_{M} x_{M}(t)+A_{C} x_{C}(t)+B_{C} d(t), \quad \text { with } t \in \mathbb{R}^{+} \backslash \mathcal{T}, \\
x_{P}(t) & =J_{P} x_{P}\left(t^{-}\right), \quad \text { with } t=t_{k}, t_{k} \in \mathcal{T}, \\
x_{M}(t) & =J_{M} x_{M}\left(t^{-}\right), \quad \text { with } t=t_{k}, t_{k} \in \mathcal{T}, \\
x_{C}(t) & =J_{C} x_{C}\left(t^{-}\right), \quad \text { with } t=t_{k}, t_{k} \in \mathcal{T}, \\
y(t) & =C_{P} x_{P}(t)-C_{M} x_{M}(t), \quad \text { with } t \in \mathbb{R}^{+} .
\end{aligned}\right.
$$

$$
\tilde{\Sigma} \equiv\left\{\begin{aligned}
\dot{x}_{P}(t) & =A_{P} x_{P}(t)+B_{P} C_{C} x_{C}(t)+B_{P} D_{C} v(t), \quad \text { with } t \in \mathbb{R}^{+} \backslash \mathcal{T}, \\
\dot{x}_{M}(t) & =\left(A_{M}+B_{M} C_{M}\right) x_{M}(t)+B_{M} v(t), \quad \text { with } t \in \mathbb{R}^{+} \backslash \mathcal{T}, \\
\dot{x}_{C}(t) & =A_{C} x_{C}(t)+B_{C} v(t), \quad \text { with } t \in \mathbb{R}^{+} \backslash \mathcal{T}, \\
x_{P}(t) & =J_{P} x_{P}\left(t^{-}\right), \quad \text { with } t=t_{k}, t_{k} \in \mathcal{T}, \\
x_{M}(t) & =J_{M} x_{M}\left(t^{-}\right), \quad \text { with } t=t_{k}, t_{k} \in \mathcal{T}, \\
x_{C}(t) & =J_{C} x_{C}\left(t^{-}\right), \quad \text { with } t=t_{k}, t_{k} \in \mathcal{T}, \\
y(t) & =C_{P} x_{P}(t)-C_{M} x_{M}(t), \text { with } t \in \mathbb{R}^{+} .
\end{aligned}\right.
$$

regulation, 53rd IEEE Conference on Decision and Control, Los Angeles, CA, USA, pp. 4697-4702.

Carnevale, D., Galeani, S. and Sassano, M. (2014b). A linear quadratic approach to linear time invariant stabilization for a class of hybrid systems, 22nd Mediterranean Conference on Control and Automation, Palermo, Italy, pp. 545-550.

Choe, D.-G. and Kim, J.-H. (2002). Pitch autopilot design using model-following adaptive sliding mode control, Journal of Guidance, Control, and Dynamics 25(4): 826-829.

Colaneri, P. and Kučera, V. (1997). The model matching problem for periodic discrete-time systems, IEEE Transactions on Automatic Control 42(10): 1472-1476.

Conte, G. and Perdon, A.M. (1995). The disturbance decoupling problem for systems over a ring, SIAM Journal on Control and Optimization 33(3): 750-764.

Conte, G., Perdon, A.M. and Zattoni, E. (2013). A geometric approach to output regulation for discrete-time switched linear systems, 21st Mediterranean Conference on Control and Automation, Platanias-Chania, Crete, Greece, pp. 852-857.

Conte, G., Perdon, A.M. and Zattoni, E. (2014). Model matching problems for switching linear systems, IFAC Proceedings Volumes 47(3): 1501-1506.

Conte, G., Perdon, A.M. and Zattoni, E. (2015a). The disturbance decoupling problem for jumping hybrid systems, 54th IEEE Conference on Decision and Control, Osaka, Japan, pp. 1589-1594.
Conte, G., Perdon, A.M. and Zattoni, E. (2015b). The disturbance decoupling problem with quadratic stability for LPV systems, IFAC-PapersOnLine 48(26): 1-6.

Conte, G., Perdon, A.M. and Zattoni, E. (2017). Unknown input observers for hybrid linear systems with state jumps, IFACPapersOnLine 50(1): 6458-6464.

Djemai, M. and Deefort, M. (2015). Hybrid Dynamical Systems, Lecture Notes in Control and Information Sciences, Vol. 457, Springer, Berlin/Heidelberg.

Engell, S., Frehse, G. and Schnieder, E. (2002). Modeling, Analysis and Design of Hybrid Systems, Lecture Notes in Control and Information Sciences, Vol. 279, Springer, Berlin/Heidelberg.

Goebel, R., Sanfelice, R.G. and Teel, A.R. (2012). Hybrid Dynamical Systems: Modeling, Stability, and Robustness, Princeton University Press, Princeton, NJ.

Haddad, W.M., Chellaboina, V.S. and Nersesov, S.G. (2006). Impulsive and Hybrid Dynamical Systems: Stability, Dissipativity, and Control, Princeton Series in Applied Mathematics, Vol. 6, Princeton University Press, Princeton, NJ.

Hua, C.-C. and Ding, S. (2011). Model following controller design for large-scale systems with time-delay interconnections and multiple dead-zone inputs, IEEE Transactions on Automatic Control 56(4): 962-968.

Kaczorek, T. (1982). Polynomial equation approach to exact model matching problem in multivariable linear systems, International Journal of Control 36(3): 531-539. 
Kouhi, Y., Bajcinca, N. and Sanfelice, R.G. (2013). Suboptimality bounds for linear quadratic problems in hybrid linear systems, 2013 European Control Conference, Zürich, Switzerland, pp. 2663-2668.

Kučera, V. (1992). Model matching of descriptor systems by proportional state feedback, Automatica 28(2): 423-425.

Lawrence, D.A. (2014). Controlled invariant subspaces for linear impulsive systems, 2014 American Control Conference, Portland, OR, USA, pp. 2336-2341.

Lawrence, D.A. (2015). Conditioned invariant subspaces for linear impulsive systems, 2015 American Control Conference, Chicago, IL, USA, pp. 4850-4855.

Li, Z., Soh, Y. and Wen, C. (2005). Switched and Impulsive Systems: Analysis, Design and Applications, Lecture Notes in Control and Information Sciences, Vol. 313, Springer-Verlag, Berlin/Heidelberg.

Lin, W.-S. and Tsai, C.-H. (1999). Neurofuzzy-model-following control of MIMO nonlinear systems, IEE Proceedings: Control Theory and Applications 146(2): 157-164.

Marro, G. and Zattoni, E. (2002). A novel geometric insight into the model matching problem with stability, 41st IEEE Conference on Decision and Control, Las Vegas, NV, USA, pp. 2137-2142.

Marro, G. and Zattoni, E. (2005). Self-bounded controlled invariant subspaces in model following by output feedback: Minimal-order solution for nonminimum-phase systems, Journal of Optimization Theory and Applications 125(2): 409-429.

Medina, E.A. and Lawrence, D.A. (2006). Controlled and conditioned invariants for linear impulsive systems, 45th IEEE Conference on Decision and Control, San Diego, CA, USA, pp. 2753-2758.

Medina, E.A. and Lawrence, D.A. (2009). State estimation for linear impulsive systems, 2009 American Control Conference, St. Louis, MO, USA, pp. 1183-1188.

Moog, C.H., Conte, G. and Perdon, A.M. (1991). Model matching and factorization for nonlinear systems: A structural approach, SIAM Journal on Control and Optimization 29(4): 769-785.

Morse, A.S. (1973). Structure and design of linear model following systems, IEEE Transactions on Automatic Control 18(4): 346-354.

Ni, M., Er, M., Leithead, W. and Leith, D. (2001). New approach to the design of robust tracking and model following controllers for uncertain delay systems, IEE Proceedings: Control Theory and Applications 148(6): 472-477.

Noldus, E. (1987). Non-linear model following, Automatica 23(3): 387-391.

Pascual, M., Garcerá, G., Figueres, E. and González-Espín, F. (2008). Robust model-following control of parallel UPS single-phase inverters, IEEE Transactions on Industrial Electronics 55(8): 2870-2883.

Perdon, A.M., Conte, G. and Zattoni, E. (2016a). Necessary and sufficient conditions for asymptotic model matching of switching linear systems, Automatica 64(2): 294-304.
Perdon, A.M., Zattoni, E. and Conte, G. (2016b). Disturbance decoupling with stability for linear impulsive systems, IFAC-PapersOnLine 49(9): 1-6.

Perdon, A.M., Zattoni, E. and Conte, G. (2016c). Model matching with strong stability in switched linear systems, Systems \& Control Letters 97(11): 98-107.

Rajasekaran, J., Chunodkar, A. and Padhi, R. (2009). Structured model-following neuro-adaptive design for attitude maneuver of rigid bodies, Control Engineering Practice 17(6): 676-689.

Savkin, A.V. and Evans, R.J. (2002). Hybrid Dynamical Systems. Controller and Sensor Switching Problems, Control Engineering Series, Birkhauser, Boston, MA.

Shioemaru, S. and Wu, H. (2001). Decentralized robust tracking and model following for uncertain large-scale interconnected systems, Journal of Optimization Theory and Applications 110(1): 35-52.

Shyu, K.-K. and Chen, Y.-C. (1995). Robust tracking and model following for uncertain time-delay systems, International Journal of Control 62(3): 589-600.

Van der Schaft, A.J. and Schumacher, H. (2000). An Introduction to Hybrid Dynamical Systems, Lecture Notes in Control and Information Sciences, Vol. 251, Springer, Berlin/Heidelberg.

Wang, D., Wu, S. and Okubo, S. (2009). Design of the state predictive model following control system with time-delay, International Journal of Applied Mathematics and Computer Science 19(2): 247-254, DOI: 10.2478/v10006-009-0020-8.

Wang, L., Zhu, J. and Park, J. (2012). A probabilistic approach for model following of Markovian jump linear systems subject to actuator saturation, International Journal of Control, Automation and Systems 10(5): 1042-1048.

Wolovich, W.A. (1972). The use of state feedback for exact model matching, SIAM Journal on Control 10(3): 512-523.

Wonham, W.M. (1985). Linear Multivariable Control: A Geometric Approach, 3rd Edn., Springer-Verlag, New York, NY.

$\mathrm{Wu}, \mathrm{H}$. (2004). Adaptive robust tracking and model following of uncertain dynamical systems with multiple time delays, IEEE Transactions on Automatic Control 49(4): 611-616.

Zakharov, A., Zattoni, E., Xie, L., Garcia, O. and Jämsä-Jounela, S.-L. (2013). An autonomous valve stiction detection system based on data characterization, Control Engineering Practice 21(11): 1507-1518.

Zakharov, A., Zattoni, E., Yu, M. and Jämsä-Jounela, S.-L. (2015). A performance optimization algorithm for controller reconfiguration in fault tolerant distributed model predictive control, Journal of Process Control 34(8): 56-69.

Zattoni, E. (2007). Decoupling of measurable signals via self-bounded controlled invariant subspaces: Minimal unassignable dynamics of feedforward units for prestabilized systems, IEEE Transactions on Automatic Control 52(1): 140-143. 
Zattoni, E. (2014). Measurable disturbance rejection with stability in continuous-time switched linear systems under dwell-time switching, European Control Conference 2014, Strasbourg, France, pp. 2242-2247.

Zattoni, E. (2016a). Disturbance decoupling with stability in discrete-time switching linear systems: Arbitrary switching, International Journal of Pure and Applied Mathematics 110(1): 227-250.

Zattoni, E. (2016b). Output feedback model matching in linear impulsive systems with control feedthrough: A structural approach, IOP Journal of Physics: Conference Series 783: $1-9$.

Zattoni, E. and Marro, G. (2013a). A constructive condition for inaccessible signal rejection with quadratic stability in discrete-time linear switching systems, 52nd IEEE Conference on Decision and Control, Florence, Italy, pp. 4650-4655.

Zattoni, E. and Marro, G. (2013b). Measurable disturbance rejection with quadratic stability in continuous-time linear switching systems, European Control Conference 2013, Zürich, Switzerland, pp. 2157-2162.

Zattoni, E., Perdon, A.M. and Conte, G. (2013a). A geometric approach to output regulation for linear switching systems, IFAC Proceedings Volumes 46(2): 172-177.

Zattoni, E., Perdon, A.M. and Conte, G. (2013b). The output regulation problem with stability for linear switching systems: A geometric approach, Automatica 49(10): 2953-2962.

Zattoni, E., Perdon, A.M. and Conte, G. (2014a). Disturbance decoupling with stability in continuous-time switched linear systems under dwell-time switching, IFAC Proceedings Volumes 47(3): 164-169.

Zattoni, E., Perdon, A.M. and Conte, G. (2014b). Output feedback model matching with strong stability in continuous-time switched linear systems, 22nd Mediterranean Conference on Control and Automation, Palermo, Italy, pp. 525-530.

Zattoni, E., Perdon, A.M. and Conte, G. (2015). Output regulation by error dynamic feedback in linear time-invariant hybrid dynamical systems, 14th European Control Conference, Linz, Austria, pp. 1438-1443.
Zattoni, E., Perdon, A.M. and Conte, G. (2016). Disturbance decoupling with closed-loop modes stability in switched linear systems, IEEE Transactions on Automatic Control 61(10): 3115-3121.

Zattoni, E., Perdon, A.M. and Conte, G. (2017a). Output regulation by error dynamic feedback in hybrid linear systems with state jumps, IFAC-PapersOnLine 50(1): 10808-10815.

Zattoni, E., Perdon, A.M. and Conte, G. (2017b). Output regulation by error dynamic feedback in hybrid systems with periodic state jumps, Automatica 81(7): 322-334.

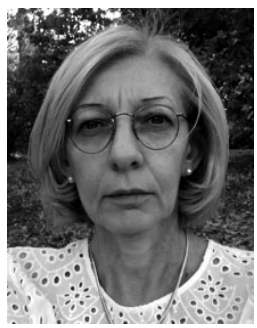

Elena Zattoni received the Laurea degree with honors in electronics engineering in 1995 and the $\mathrm{PhD}$ in systems engineering in 1999 from the University of Bologna, Italy. She has been an assistant professor at the University of Bologna since 2001. She has been a visiting professor at Aalto University, the University of Michigan, IRCCyN, and Brown University. Her research interests are focused on structural approaches to automatic control, with special emphasis on the synthesis of enhanced-reliability control systems. She has authored more than 150 papers in the field. She is a member of the IFAC TC on Linear Control Systems. She is a senior member of the IEEE and has served in the Member Activity Board of the IEEE Control Systems Society.

Received: 30 January 2017 Revised: 25 April 2017

Accepted: 30 June 2017 